\title{
El Uso de Las Letras como Fuente de Errores de Estudiantes Universitarios en la Resolución de Tareas Algebraicas
}

\author{
Use of Letters as a Source of Errors for University Students in Solving
}

Algebraic Tasks

\author{
José García Suárez \\ Isidoro Segovia Alex ${ }^{* *}$ \\ José Luis Lupiáñez Gómez ${ }^{* * *}$
}

\begin{abstract}
Resumen
La presente investigación es un estudio realizado con 194 estudiantes del Centro Universitario de la Costa Sur, en Autlán, México, cuyo objetivo es analizar los errores más comunes que los alumnos de primer semestre presentan en las producciones, al operar con los distintos significados que pueden tener las letras en álgebra y con base a esos resultados, establecer su ubicación dentro de alguna de las cuatro categorías de entendimiento en el uso y significado de las letras en álgebra que propone Küchemann (1980). Los resultados muestran que más de la mitad de los estudiantes de este nivel educativo no manifiestan dificultades al evaluar las letras, manejarlas como objetos o considerar su presencia, sin embargo, sí revelan deficiencias en el discernimiento para comprender el uso y significado de las letras como incógnitas de valor especifico, números generalizados y como variables.
\end{abstract}

Palabras-clave: Errores. Tareas Algebraicas. Usos de las Letras. Niveles de Entendimiento. Estudiantes Universitarios.

\begin{abstract}
The present investigation is a study with 194 students of the Centro Universitario de la Costa Sur in Autlan, Mexico. It aims to analyze the most common mistakes that first semester students show in the productions, to operate with different meanings that may have letters in algebra and, based on these results, establish its location within one of the four categories of understanding in the use and meaning of letters in algebra proposed by Küchemann (1980). The results show that more than half of the students at this level of education do not show difficulties in evaluating letters, handling them as objects or considering their presence, however, it does reveal deficiencies in the discernment to understand the use and meaning of the letters as unknown of specific value, numbers, and variables such as widespread.
\end{abstract}

Keywords: Errors. Algebraic Tasks. Uses of Letters. Levels of Understanding. University Students.

\section{Introducción}

\footnotetext{
* Master Oficial de Didáctica de la Matemática por la Universidad de Granada (UGR). Profesor Docente del Centro Universitario de la Costa Sur de la Universidad de Guadalajara, México (CUCSUR). Dirección postal: Casimiro Castillo 19, C.P. 48900, Autlán, Mexico.Email: josegar@cucsur.udg.mx

** Doctor en Didáctica de la Matemática por la Universidad de Granada (UGR). Profesor Titular en el Departamento de Didáctica de la Matemática. Universidad de Granada (UGR). Granada, España. Dirección postal: Facultad de Educación, Campus de Cartuja, 18071 Granada, España. E-mail: isegovia@ugr.es

** Doctor en Didáctica de la Matemática por la Universidad de Granada (UGR). Profesor Titular en el Departamento de Didáctica de la Matemática. Universidad de Granada (UGR). Granada, España. Dirección postal: Facultad de Educación, Campus de Cartuja, 18071 Granada, España. E-mail: lupi@ugr.es
} 
Históricamente la enseñanza y aprendizaje del álgebra no han producido los resultados deseados en la formación educativa de los estudiantes de educación secundaria y bachillerato (BOOTH, 1981, 1988; KÜCHEMANN, 1978, 1980; URSINI; TRIGUEROS, 2004; USISKIN, 1988; RUANO; SOCAS; PALAREA, 2008; HODGEN et al., 2009). En la actualidad, esta situación no ha cambiado y se refleja en el desempeño de aquellos estudiantes, quienes al ingresar a la universidad manifiestan un deficiente aprendizaje, con nociones pobres acerca de conceptos algebraicos elementales, exhibiendo un cúmulo de ideas confusas que difícilmente asimilan o retienen, provocando dificultades en el entendimiento de nociones y conceptos básicos del álgebra (LINCHEVSKI, 1995; POCHULU, 2005; CAPUTO; MACIAS, 2006; FERREYRA et al., 2010). Esta situación puede tener importantes repercusiones cuando los estudiantes se enfrentan con asignaturas de matemáticas cuyos contenidos tienen un mayor nivel de complejidad cognitiva y que pueden tener mucha importancia en su desarrollo profesional posterior.

En lo que respecta a investigaciones relacionadas con la enseñanza y aprendizaje del álgebra en niveles educativos superiores en México, consideramos aportaciones importantes los trabajos de Backhoff y Tirado (1993), quienes afirman que, aproximadamente, más de la mitad de los estudiantes universitarios participantes en su investigación, tienen problemas con el dominio de los conocimientos básicos de álgebra que se imparten en secundaria (comprensión del despeje de incógnitas, evaluación de expresiones algebraicas, simplificación de ecuaciones algebraicas lineales, solución de ecuaciones de primer grado y planteamiento y solución de problemas en términos de ecuaciones). Así mismo, Ursini y Trigueros (2006 realizaron estudios orientados a evaluar el domino del manejo de la variable en estudiantes universitarios y mencionan, en sus resultados, que la mayoria de los sujetos analizados sólo son capaces de integrar los usos de las letras como número general y como incognita cuando se les presentan problemas sencillos. Muchos de ellos tampoco son capaces de simbolizar expresiones, ni interpretar el papel de las variables involucradas en el problema, ni establecer o manejar las relaciones entre las variables.

Por otra parte, Usiskin (1988) señaló que en el aprendizaje del álgebra, un tema de gran dificultad ha sido, resolver tareas algebraicas centradas en las operaciones básicas (sumas, restas, multiplicaciones y divisiones de expresiones algebraicas), en la formulación y solución de ecuaciones, así como el manejo de las funciones. Küchemann (1980), al respecto, estableció que las razones de estas dificultades pueden residir en los diferentes usos que los estudiantes dan a las letras cuando éstas se presentan en distintos contextos, manifestando en 
muchas ocasiones su incapacidad de distinguir sus significados. Diversos trabajos de investigación han sido enfocados a los usos de las letras en álgebra y su relación con el concepto de variable, entre ellos Collis (1975), que documenta las diversas maneras que los estudiantes interpretan las letras empleadas como símbolos matemáticos. Tomando como base este trabajo, Küchemann (1980), identifica seis tipos de usos de las letras en las matemáticas escolares de nivel secundaria: letra evaluada, letra no utilizada o ignorada, letra como objeto, letra como incógnita, letra como número generalizado y letra como variable, que constituyen el referente que usaremos en la parte empírica de nuestro estudio.

En este trabajo queremos constatar el bajo nivel de conocimientos algebraicos que los estudiantes tienen al ingresar a la universidad a través de los errores más comunes y que no corresponden a su grado académico. Así, el objetivo de nuestro estudio es detectar, analizar y organizar los errores sistemáticos que tienen los estudiantes universitarios cuando abordan tareas algebraicas que requieren el conocimiento y el manejo de distintos usos de las letras y relacionar las dificultades que los originan con sus niveles de comprensión en álgebra. Para esto se consideró el test de evaluación de Concepts in Secondary Mathematics and Science Study in Álgebra (CSMS), desarrollado en Inglaterra por el King's College London, en 1980, actualizado en 2008 y modificado y adaptado al contexto de México. A partir de esta indagación, revelamos cómo los problemas de aprendizaje no resueltos del álgebra escolar en niveles educativos obligatorios, en México, tienen incidencias aún en el nivel terciario de formación.

Muchos trabajos indagan sobre las dificultades de niños de escuela elemental al usar álgebra, mientras que otros muestran formas de ayudar a los estudiantes para facilitar la transición curricular entre la aritmética y el álgebra. Sin embargo, pocos estudios se enfocan en mostrar que las dificultades que los estudiantes padecen en los niveles primario y secundario persisten cuando éstos inician sus estudios superiores.

Por esta razón, consideramos que la relevancia de esta investigación radica en documentar las dificultades que presentan los estudiantes en el primer curso de matemáticas en la Universidad; de esa manera, podemos establecer un marco interpretativo importante para todas aquellas instituciones educativas de nivel superior en las cuales el primer curso de matemáticas se compone, esencialmente, de contenidos algebraicos. Estudios de este tipo pueden sugerir dónde reforzar el conocimiento algebraico en aquellos estudiantes que, a pesar de contar con una formación algebraica previa, muestran carencias en esos conocimientos.

\section{Antecedentes y Marco teórico}


Diversos investigadores han manifestado que la dificultad de la significación ${ }^{1}$ de las letras, no es un algo trivial para un buen número de estudiantes. Esta situación fue constatada por Kieran (1990, p.96) quien ejemplifica esta dificultad a través del siguiente ejemplo: "En aritmética, $12 \mathrm{~m}$ puede significar 12 metros, es decir, 12 veces 1 metro. Pero en álgebra, $12 \mathrm{~m}$ puede significar 12 veces un número indeterminado de metros". Por tanto, la letra tiene dos significados diferentes según el contexto. Davis (1975) proporcionó otro dilema en el uso de la misma expresión para expresar dos cosas diferentes en el mismo contexto: $a+b$ representa a la vez, el procedimiento de añadir $a$ y $b$ y el resultado de la suma; esto se caracteriza como el dilema proceso-producto. El mismo Davis sostiene que en álgebra no hay una distinción clara entre esas dos entidades. Para el mejor entendimiento del uso de las letras; Philipp propuso siete categorías de agrupación y describe con ejemplos los distintos usos de las mismas:

Letras como etiquetas, como: $f$ e $y$ en $3 f=1 y$. En donde $f$ se usa para denotar pies e y para denotar yarda; como constantes $\pi, e$, y $c$; como incógnitas para denotar $x$ en $5 x-9=11$; como números generalizados para denotar $a$, $b$ en $a+b=b+a$; como cantidades variables para denotar $x, y$ en $y=9 x-2$; como parámetros para denotar $m, b$ en $y=m x+b$, y como símbolos abstractos para denotar $e, x$ en $e^{x}=x$. ( Philipp, 1992, p.160)

La variedad de significados que una sola letra puede tomar indica la complejidad que tiene para los estudiantes, especialmente cuando éstos intentan identificarlas y utilizarlas en diferentes contextos.

Mac Gregor y Stacey (1997) afirmaron que la mayoría de los estudiantes, hasta los 15 años, no pueden interpretar letras como números generalizados o incluso como incógnitas con valor específico; generalmente ignoran las letras y las reemplazan con valores numéricos, o consideran las letras como etiquetas, es decir, abreviaturas de las características de los objetos (iniciales de nombres de personas, de objetos o lados de figuras geométricas, entre otras). Afirmaron, también, que la principal explicación para este tipo de error tiene vínculo directo con los niveles de desarrollo cognitivo; sin embargo, proporcionaron explicaciones alternativas para orígenes específicos de interpretaciones erróneas que no han tenido demasiada repercusión en la literatura, ya que pueden o no estar asociados con el nivel cognitivo. Según ellos, estos orígenes son supuestos intuitivos y de razonamiento pragmático acerca de una nueva notación, las analogías con los sistemas de símbolos familiares, la

\footnotetext{
${ }^{1}$ Palarea (1999) define significación de las letras como: el uso y significado que los estudiantes les dan a las letras en el álgebra.
} 
interferencia del nuevo aprendizaje en matemáticas y los efectos de los materiales didácticos mal elaborados.

Un análisis más detallado acerca del uso de la letra en el álgebra, lo encontramos en Küchemann (1980), quien realizó investigaciones que involucraban a estudiantes ingleses entre 15 y 18 años que cursaban sus estudios de secundaria, clasificando los usos de las letras de la siguiente manera:

1. Letra evaluada. Es la asignación de valores numéricos arbitrarios a las letras; por ejemplo ante la cuestión, Si $e+f=8$; $e+f+g=$ ? algunos estudiantes expresan como resultado, $e+f+g=12$, asignándole valores arbitrarios de 4 a cada una de las incognitas, $4+4+4=12$.

2. Letra ignorada. En expresiones como $2 x+3 y+7 z$, algunos estudiantes dan como respuesta $12 x y z$; en este caso, los estudiantes trabajan realizando las operaciones aritmeticas $(2+3+7)$, ignorando la presencia de las letras o, en el mejor de los casos, las consideran, pero no tienen significado para ellos.

3. Letra como un objeto. Las letras pueden ser utilizadas como abreviaturas de nombres de objetos o como objetos en sí mismos, por ejemplo: $4 p+3 e$, podría representar el enunciado 4 profesores y 3 estudiantes.

4. Letra como una incógnita específica. Los estudiantes perciben que las letras tienen un valor específico pero desconocido. Por ejemplo, la expresión $E+B+C$ nunca será igual a $E+D+C$, porque $B$ no es igual a $D$ (aunque ambas sean reconocidas como variables que deben ser siempre con valor diferente una de la otra), ya que son representadas por diferentes letras del alfabeto.

5. Letras como números generales. Los estudiantes perciben que las letras representan valores, o por lo menos son capaces de tomar varios valores en lugar de sólo uno. Por ejemplo, los estudiantes, si se les pide que elaboren una lista de todos los valores posibles para la expresión $a+b=20$, presentan generalmente una lista con varios números enteros que satisfacen la condición. Sin embargo, tienden a no darse cuenta de que deben expresar, de manera obligatoria, todos los números que satisfacen la condición.

6. La letra como variación de cantidad (variable). Aquí los estudiantes ven las letras como la representación de un rango de valores no especificados, incluyendo todos los números racionales e irracionales. Los estudiantes también comprenden que la variable se define por su relación con otros términos en la expresión. Por ejemplo, en la expresión $f+g=10$ el valor de $f$ es dependiente del valor de $g$. 
En el mismo estudio, Küchemann (1980, p.70) identifica las primeras tres categorías de los usos de la letra (evaluada, ignorada y como objeto) en el nivel más elemental del manejo de ésta. En cambio, cuando los participantes reconocen las variables como incógnitas específicas, números generales o variación de cantidades, considera que tienen un entendimiento alto sobre el concepto de la misma. En este mismo sentido, sostiene que un estudiante habrá comprendido perfectamente el uso de los símbolos literales en álgebra cuando sea capaz de trabajar con la "letra como variable". Sugiere también, que es más fácil para el estudiante trabajar con la "letra como incógnita específica" que con la "letra como número generalizado", y que es más fácil trabajar con la "letra como número generalizado" que con la "letra como variable".

A partir de estas consideraciones, Küchemann formula la relación existente entre los distintos usos de las letras y caracteriza cuatro niveles de entendimiento de estudiantes en relación a su significado y su uso.

Nivel 1: en el primer nivel se resuelven las tareas puramente numéricas o que tienen una estructura algebraica simple (sustituciones numéricas directas en expresiones algebraicas en donde las literales no tienen coeficientes, multiplicación de datos numéricos, simplificación de términos semejantes que involucran una sola letra como incógnita). Los estudiantes en este nivel son capaces de resolver problemas mediante el uso de las letras como objetos (lados de figuras geométricas, iníciales de nombres de objetos) o sin usarlas en absoluto. Cuando a estos estudiantes se les presentan letras como incógnitas específicas, tienden a evaluarlas o no utilizarlas para resolver dichas situaciones. Un ejemplo de tarea propia de este nivel es: Si $a+$ $5=8$ entonces $a=\ldots$, donde la letra puede ser evaluada directamente con un valor numérico que cumpla la igualdad.

Nivel 2: la diferencia con el anterior, es la mayor familiaridad con la notación algebraica que les permite a los estudiantes resolver tareas de más complejidad. Por ejemplo, en la tarea: Si m $=3 n+1$ y $n=4$ entonces $m=\ldots$, los estudiantes deben hacer frente a una expresión literal ambigua antes de poder evaluar una letra. En este nivel existe una mayor disposición para aceptar respuestas que aparecen incompletas o ambiguas.

Nivel 3: en este nivel las letras son usadas como incógnitas de valor específico, que representan números en lugar de objetos; sin embargo, los estudiantes en este nivel sólo pueden manejar las incógnitas que presentan estructuras algebraicas simples. Por ejemplo, cuando se les solicita sumar 4 a $3 n$, no encuentran dificultad, pero al intentar resolver tareas en las que sea necesario multiplicar 4 por $n+5$, son incapaces de efectuar de manera correcta las 2 operaciones necesarias. 
Nivel 4: los estudiantes de este nivel pueden hacer frente a contextos en los cuales se requiere, como mínimo, que las letras sean consideradas como incógnitas específicas, pero donde hay una fuerte tendencia a tratar las letras como objetos, como se indica en esta otra tarea: Si los pasteles cuestan p centavos cada uno y las empanadas e centavos cada una ¿Qué significa $4 p$ $+3 e$ ? En este caso, los estudiantes deben ser capaces de distinguir que las letras no son etiquetas de las características propias de los objetos (iniciales) e inferir que la expresión enunciada puede representar distintos valores. Así mismo, este nivel involucra el manejo de las letras como números generalizados y como variables, como muestra la siguiente: ¿Cuál es más grande $2+n$ o $2 n$ ? Los estudiantes considerados dentro de este nivel de entendimiento deben ser capaces de expresar y justificar la respuesta a este ítem de manera generalizada y no limitarse a la evaluación de sólo algunos valores numéricos que satisfagan la expresión. Por esta razón, este nivel es considerado el de mayor exigencia cognitiva descrito por Küchemann.

Usiskin (1988) sostiene que el uso de las letras puede aplicarse en diferentes contextos y con distintos significados en cada uno de ellos y, dependiendo del contexto en el que se presentan, son tratadas de diferente manera; sostiene que el entendimiento del concepto de variable es el de mayor nivel de entendimiento del uso de las letras, pues implica la posibilidad de superar la simple realización del cálculo y operaciones con letras o con símbolos, para alcanzar un entendimiento de las razones por las que funcionan estos procedimientos, la capacidad de prever hacia dónde conducen y la posibilidad de establecer relaciones entre los aspectos que asume la variable. De esta forma, destaca varias asociaciones de uso de las letras a diferentes concepciones del álgebra en el cual se utilizan. Por ejemplo, una letra puede representar una incógnita específica, esto es, un número desconocido pero específico que puede ser calculado considerando las restricciones dadas; como número generalizado, es decir, un número indeterminado comprendido dentro de un método general; o ser utilizado para representar una relación funcional entre dos cantidades cuyos valores cambian. Más aún, puede utilizarse de diferentes formas en momentos distintos dentro de un mismo problema, asumiendo distintas caracterizaciones. Estas capacidades son esperadas en alumnos de primer curso universitario, como con quienes se llevó a cabo esta investigación.

\section{Descripción metodológica}


Nuestra investigación responde a un estudio descriptivo de carácter cuantitativo y cualitativo, dirigido al análisis del uso de las letras en álgebra y los errores manifestados por los estudiantes de primer curso universitario al resolver distintas tareas algebraicas. Los participantes fueron en total 194 estudiantes de primer curso universitario, del Centro Universitario de la Costa Sur de la Universidad de Guadalajara, México, cuyas edades oscilaron entre 18 y 20 años. Los alumnos participantes fueron elegidos por el nivel educativo en el que estaban inscritos en el momento de realizar este trabajo.

El desarrollo del estudio se llevó a cabo entre septiembre y diciembre de 2012 y se basó en la categorización de los distintos usos de las letras propuestos por Küchemann (1980), con el fin de caracterizar la forma en la que los estudiantes interpretan los símbolos literales en diferentes contextos algebraicos. Para ello se aplicó un cuestionario escrito en el que los alumnos deben interpretar y manipular expresiones algebraicas, así como resolver problemas representados por símbolos literales. Este instrumento está basado en el modelo del proyecto Concepts in Secondary Mathematics and Science Study in Álgebra referido en la introducción, y para su mejor comprensión por los estudiantes, se realizaron modificaciones en el lenguaje para adaptarlo al contexto social mexicano en el cual se implementó.

El instrumento, originalmente diseñado por Küchemann, Brown y Blackeley (KÜCHEMANN, 1980, p.16), está compuesto por 23 tareas en torno de distintos contenidos algebraicos: sustitución formal, simplificación, generalización y formulación, interpretación y solución de ecuaciones ${ }^{2}$. Se aplicó en sesiones de 50 minutos y, una vez analizadas las respuestas, se realizaron entrevistas semi-estructuradas (HERNÁNDEZ; FERNÁNDEZ; BAPTISTA, 1999) a una muestra de 10 estudiantes, con el objetivo de profundizar en la búsqueda de las fuentes de los errores mediante las respuestas expresadas de manera incorrecta.

\subsection{Las tareas}

Las tareas fueron diseñadas por Küchemann para cada categoría, describiéndose cada una de ellas en función de la respuesta esperada, es decir, distinguiendo cuándo los estudiantes tenían que interpretar letras en cada ítem como valores evaluados, objetos, incógnitas con valor específico, números generalizados y variables. La evaluación de la

\footnotetext{
${ }^{2}$ Es posible consultar el cuestionario a través del enlace: http://goo.gl/MSQIXQ.
} 
prueba fue organizada de acuerdo con los niveles de entendimiento en el manejo de las letras de este autor, bajo el siguiente formato:

Letra evaluada. Se asigna a la letra un valor numérico.

Letra ignorada. Se ignora la letra presente o al menos se reconoce su existencia sin darle significado.

Letra como objeto. Se considera la letra como una forma abreviada de un objeto o como objeto en sí mismo con valor propio.

Letra como incógnita de valor específico. Se considera una letra como número único, pero desconocido y que pueden operar directamente sobre ella.

Letra como número generalizado. La letra es vista como una incógnita que puede tomar varios valores

Letra como variable. Las letras son la representación de un rango de valores desconocidos y con una relación sistemática existente entre estos conjuntos de valores.

Las tareas relacionadas con cada uno de los niveles de entendimiento, que fueron consideradas para el análisis de las respuestas de la prueba aplicada se presentan en el Cuadro 1 y son las misma que propone Küchemann (1980).

\begin{tabular}{cl}
\hline Nivel & \multicolumn{1}{c}{ Criterio } \\
\hline 0 & Menos de 4 respuestas correctas de las tareas del nivel 1 \\
1 & $\mathrm{Al}$ menos 4 de 6 respuestas correctas de las tareas 5(a), 6(a), 7(b), 8, 9(a), 13(a). \\
2 & $\mathrm{Al}$ menos 5 de 7 respuestas correctas de las tareas 7(c), 9(b), 9(c), 11(a), 11(b), 13(d), 15(a). \\
3 & $\mathrm{Al}$ menos 5 de 8 respuestas correctas de las tareas 4(c), 5(c), 9(d), 13(b), 13(h), 14, 15(b), 16. \\
4 & $\mathrm{Al}$ menos 6 de 9 respuestas correctas de las tareas 3, 4(e),7(d),17(a),18(b),19, 20, 21, 22. \\
\hline
\end{tabular}

\section{Cuadro 1 - Tareas y niveles de entendimiento}

Fuente: Küchemann (1980, p.64-69)

La prueba se aplicó en las primeras semanas del ciclo escolar y se seleccionaron las carreras de Licenciatura en Nutrición, Licenciatura en Administración de Empresas, Técnico Superior en Electrónica y Mecánica Automotriz, Ingeniería en Procesos y Comercio Internacional, Ingeniería en Teleinformática e Ingeniería en Recursos Naturales y Agropecuarios.

\section{Resultados y discusión}

En este apartado presentamos y discutimos los resultados obtenidos mediante la aplicación del cuestionario. En primer lugar se fundamentan las razones por las cuales se ubicaron a los estudiantes en los distintos niveles de entendimiento tomando como referencia principal el marco propuesto por Küchemann (1980). A continuación, detallamos algunas 
respuestas significativas, que nos llevaron a situar a los estudiantes participantes en este estudio en los distintos niveles antes mencionados. Al mismo tiempo, consideramos que el análisis de los errores en las producciones de los estudiantes, junto con las entrevistas realizadas, aportaron evidencias importantes sobre las probables fuentes de los mismos, lo cual permitió confirmar cierta similitud de los errores informados en este estudio con aquellos errores reseñados en la literatura consultada.

\subsection{Designación de los estudiantes en los diferentes niveles de entendimiento}

Con el objetivo de explicar el método empleado para ubicar a los estudiantes en los respectivos niveles de entendimiento, describiremos como ejemplo el análisis de las respuestas del estudiante número 1, situado en el primer nivel (Tabla 1):

Tabla 1 - Respuestas correctas requeridas para el nivel 1

\begin{tabular}{lcccccc}
\hline & \multicolumn{7}{c}{ Tareas } \\
\cline { 2 - 7 } \# Estudiante & $5 \mathrm{a}$ & $6 \mathrm{a}$ & $7 \mathrm{~b}$ & 8 & $9 \mathrm{a}$ & $13 \mathrm{a}$ \\
\hline 1 & $\checkmark$ & $\checkmark$ & $\checkmark$ & $\checkmark$ & $\mathrm{x}$ & $\mathrm{x}$ \\
\hline
\end{tabular}

La tabla 1 muestra que el estudiante contesta correctamente ( $\square$ ) a cuatro tareas e incorrectamente $(\mathrm{x})$ a dos de las diseñadas para el nivel 1. Es decir, responde correctamente al menos a 4 de las 6 tareas de ese nivel, cumpliendo con suficiencia la condición requerida en el Cuadro 1. Para completar nuestra valoración, evaluamos las respuestas a las tareas consideradas en el nivel 2, no cumpliendo con el número mínimo de respuestas correctas de las tareas requeridas de ese nivel, 5 de 7, como muestra la tabla 2.

Tabla 2 - Respuestas correctas requeridas para el nivel 2

\begin{tabular}{cccccccc}
\hline & \multicolumn{7}{c}{ Tareas } \\
\cline { 2 - 8 } \# Estudiante & $7 \mathrm{c}$ & $9 \mathrm{~b}$ & $9 \mathrm{c}$ & $11 \mathrm{a}$ & $11 \mathrm{~b}$ & $13 \mathrm{~d}$ & $15 \mathrm{a}$ \\
\hline 1 & $\checkmark$ & $\mathrm{x}$ & $\mathrm{x}$ & $\checkmark$ & $\mathrm{x}$ & $\mathrm{x}$ & $\checkmark$ \\
\hline
\end{tabular}

De manera similar se procedió con el total de las pruebas analizadas con el objetivo de categorizar a todos los estudiantes. A continuación, reseñaremos los resultados obtenidos, distinguiendo las respuestas de los estudiantes ubicados en cada nivel de entendimiento. 


\subsection{Resultados particulares del nivel 1 de entendimiento}

La tabla 3 muestra los resultados del análisis de las respuestas de los estudiantes ubicados en este primer nivel de entendimiento ${ }^{3}$ a los ítems del nivel 2. Se presentan el número de estudiantes que no dieron respuestas a determinadas tareas, la cantidad de respuestas correctas y la e respuestas incorrectas. Consideramos conveniente recordar que de acuerdo con la metodología establecida por Küchemann (1980), se analizan las respuestas erroneas del nivel inmediato superior, las cuales imposibilitan ubicar a los estudiantes en ese nivel de entendimiento. Por último, en la parte inferior se presenta el análisis de las respuestas incorrectas que se observaron en las producciones de los estudiantes de ese nivel. Las respuestas analizadas nos llevaron a situar en él al 25.6\% del total de la muestra.

Tabla 3 - Estudiantes del nivel 1: tipos de respuesta y usos de las letras

\begin{tabular}{llllllll}
\hline & \multicolumn{7}{c}{ Ítem } \\
\cline { 2 - 7 } Tipo de respuesta & $7 c$ & $9 b$ & $9 c$ & $11^{a}$ & $11 b$ & $13 d$ & $15 a$ \\
Sin respuesta & & & & & & & \\
Correctas & 19 & 7 & 10 & 20 & 22 & 4 & 12 \\
Incorrectas & 17 & 34 & 28 & 24 & 12 & 28 & 13 \\
& 14 & 9 & 12 & 6 & 16 & 18 & 25 \\
Uso de las letras & & & & & & & \\
Evaluada & 2 & 2 & 7 & $\checkmark$ & $\checkmark$ & 0 & 25 \\
Ignorada & 12 & 7 & 5 & 0 & 4 & 18 & 0 \\
Como objeto & 0 & 0 & 0 & 0 & 0 & $\checkmark$ & 0 \\
Incógnita de valor especifico & $\checkmark$ & 0 & 0 & 2 & 9 & 0 & $\checkmark$ \\
Número generalizado & 0 & $\checkmark$ & $\checkmark$ & 4 & 3 & 0 & 0 \\
\hline
\end{tabular}

Como se puede observar en la tabla 3 , el ítem 15(a), presentó la mayor frecuencia de respuestas erróneas por parte de los estudiantes ubicados en el nivel 1. El 74\% de los estudiantes respondieron de manera incorrecta el citado ítem, aplicando un procedimiento mediante el cual sustituían los datos numéricos dados en el enunciado del problema para intentar resolverlo.

La Figura 1 muestra un ejemplo prototípico de las respuestas que fueron consideradas dentro de este primer nivel de entendimiento para el caso de los ítems de la tarea 9.

\footnotetext{
${ }^{3}$ En las tablas de resultados emplearemos el símbolo $(\checkmark)$ para indicar cuál es el uso de la letra esperado como respuesta correcta en cada una de las tareas analizadas.
} 


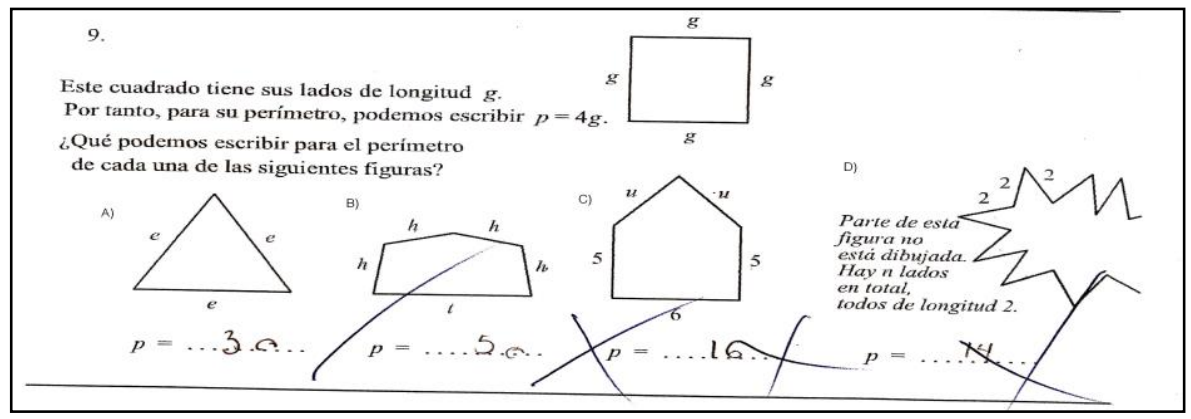

Figura 1 - Respuestas del estudiante número 5 a la tarea 9

En la figura 1 se pueden apreciar las respuestas de dos de los usos más habituales que los estudiantes de este nivel atribuyen a las letras: letra como objeto y letra ignorada. En el ítem 9 (b), el estudiante responde que el perímetro de la figura es $p=5 e$. En este caso el estudiante identifica las letras presentes como objetos (los lados de la figura), asociando a la letra $e$ un valor desconocido, según manifestó el estudiante en la entrevista posterior. Por otra parte, se puede advertir en el ítem 9 (c), que el mismo estudiante ignora las letras presentes al afirmar que el perímetro de esa figura es: $p=16$.

Algunas otras respuestas incorrectas frecuentes exhibían diversas deficiencias en los conocimientos no sólo algebraicos, sino aritméticos, pues encontramos respuestas erróneas de sumas y multiplicaciones aritméticas en las respuestas de algunas tareas. Así mismo, en algunas otras respuestas se advirtió la tendencia de los estudiantes a responder correctamente las expresiones que les permitían resolver una expresión con una sola operación y se mostraban incapaces de resolver aquellas que les exigían más de dos operaciones o relacionar varias de éstas, evidenciando, de esta forma, deficiencias en sus conocimientos algebraicos elementales. En algunos casos, los estudiantes son incapaces de resolver una suma algebraica, como la incluida en el ítem 13 (a), del primer nivel - Escribe de manera sencilla la siguiente expresión: $2 a+5 a=$ ?... obteniendo respuestas como 7 o $7 a^{2}$.

\subsection{Resultados particulares del nivel 2 de entendimiento}

Los resultados sitúan en este nivel al 53.8\% de los estudiantes, que fueron los que resolvieron de manera correcta las tareas propias de los dos primeros niveles, pero en cambio, no superaron las propias del nivel 3. La tabla 4 muestra los resultados del análisis de las 
respuestas de los estudiantes ubicados en este segundo nivel de entendimiento respecto a los ítems del nivel 3.

Tabla 4 -Estudiantes del nivel 2: tipos de respuesta y usos de las letras

\begin{tabular}{lllllllll}
\hline & \multicolumn{7}{c}{ Ítem } \\
\cline { 2 - 8 } Tipo de respuesta & $4 \mathrm{c}$ & $5 \mathrm{c}$ & $9 \mathrm{~d}$ & $13 \mathrm{~b}$ & $13 \mathrm{~h}$ & 14 & $15 \mathrm{~b}$ & 16 \\
\cline { 2 - 8 } Sin respuesta & 3 & 19 & 23 & 10 & 10 & 29 & 22 & 17 \\
Correctas & 35 & 25 & 30 & 38 & 60 & 29 & 27 & 11 \\
Incorrectas & 66 & 60 & 51 & 56 & 34 & 46 & 55 & 76 \\
\hline Uso de las letras & & & & & & & & \\
Evaluada & 38 & 39 & 28 & 0 & 0 & 31 & 11 & 0 \\
Ignorada & 26 & 0 & 0 & 4 & 21 & 0 & 0 & 0 \\
Como objeto & 0 & 17 & 23 & 52 & 13 & 10 & 41 & 3 \\
Incógnita de valor especifico & $\checkmark$ & 2 & 0 & 0 & 0 & $\checkmark$ & 0 & 60 \\
Número generalizado & 0 & $\checkmark$ & $\checkmark$ & $\checkmark$ & $\checkmark$ & 0 & $\checkmark$ & $\checkmark$ \\
Otros & 2 & 2 & 0 & 0 & 0 & 5 & 3 & 13 \\
\hline
\end{tabular}

La tabla 4 destaca cómo la tarea que presentó mayor frecuencia de respuestas incorrectas fue la 16 (Figura 2), ya que más del $73 \%$ de los estudiantes de este nivel expresaron respuestas utilizando las letras como incógnitas de valor especifico en lugar de considerarlas como números generalizados. Sobresale, también, la frecuencia elevada de respuestas incorrectas encontradas en el ítem 13 (b), que presentó una alta frecuencia de respuestas erróneas en los estudiantes ubicados en el nivel 2 (53.8\%), utilizando la letra como objeto, la mayoría de ellos son incapaces de aceptar una respuesta que incluya letras con el significado de incógnitas.

La figura 2 muestra otro ejemplo típico encontrado en las respuestas de los estudiantes de este nivel.

$$
\text { 16. Qué puedes decir sobre de } c \text { si } \begin{aligned}
& c+d=10 \\
& \\
& \text { y }
\end{aligned}
$$

Figura 2 - Respuestas del estudiante número 81 a la tarea 16

En este caso, el estudiante empleó la letra como una incógnita de valor especifico, es decir, asigna un valor que satisface la condición dada en el enunciado del problema, y acepta ese valor como respuesta válida sin considerar que puede incluir un rango determinado de valores conocidos (números generalizados). Para profundizar en esta consideración, se entrevistó al alumno: 
Investigador: Al observar tu respuesta al ítem 16, no me queda claro como la obtienes. ¿Me puedes comentar acerca de cómo obtienes que c $=4$ y d=10 como respuesta?

Estudiante: Como dice que c y d, deben sumar 10, entonces dividí 10 entre 2 y me dio 5. Después dice que c es menor que d, por lo que des 6 y c debe ser 4.

I: ¿El resultado que obtuviste, satisface todos los casos posibles de respuestas que pueden presentarse de la expresión algebraica enunciada?

E: No entiendo, ¿Cuales casos posibles?

I: Es decir, ¿Puede haber otras parejas de números que sumados den 10 y que uno sea mayor que el otro?

E: Oh si, por ejemplo 3 y 7 .

I: Así es, otros ejemplos 2 y 8, 1 y 9. ¿Mantienes la misma respuesta, después de las observaciones que hicimos?

E:Ummm...no, entonces ¿c puede tener más valores aparte del 4?

I: Así es, y como deben ser esos valores, observa los ejemplos. ¿Cuánto valía c en los ejemplos que dimos?

E: $4,3,2,1 \ldots$

I: ¿Cómo puedes expresar el valor de c, de acuerdo a esos ejemplos?

E: Quec puede ser 4 o menor que 4.

I: Correcto, finalmente para este problema, ¿Puedes decirme porque considerabas que la incógnita sólo tenía un valor?

E: Pues, porque al encontrar un valor que la resolvía no busque más, al pensar que con eso era suficiente...

Este análisis nos permite inferir que los estudiantes ubicados dentro de este nivel manejan adecuadamente las letras en estructuras algebraicas con mayor dificultad cognitiva que las consideradas en el nivel 1. Sin embargo, persiste su tendencia a evaluar las letras cuando desconocen el procedimiento correcto para resolver una determinada tarea. Por otro lado, muestran deficiencias para comprender que las letras pueden representar números generalizados y variables. Finalmente, se percibe en las respuestas de estos estudiantes una mayor disposición para aceptar respuestas que parecen incompletas o ambiguas, lo cual puede deberse a una mayor familiaridad con la notación algebraica.

\subsection{Resultados particulares del nivel 3 de entendimiento}

En este nivel se incluyen aquellos estudiantes que pueden operar las letras como incógnitas de valor específico, es decir, las letras ahora representan números en lugar de objetos. Sin embargo, estos estudiantes sólo pueden manejar las incógnitas que presentan estructuras algebraicas simples y siguen encontrando dificultades para realizar las operaciones que implican el manejo de estructuras algebraicas más complejas.

Los resultados obtenidos del análisis en las respuestas nos indicaron que el $17.9 \%$ de los estudiantes quedaron ubicados en este nivel de acuerdo a los criterios establecidos en el Cuadro 1. La tabla 5 muestra el resumen de resultados respecto a los ítems del nivel 4. 
Tabla 5 - Estudiantes del nivel 3: tipos de respuesta y usos de las letras

\begin{tabular}{|c|c|c|c|c|c|c|c|c|c|}
\hline \multirow{3}{*}{ Tipo de respuesta } & \multicolumn{9}{|c|}{ Ítem } \\
\hline & 3 & $4 \mathrm{e}$ & $7 d$ & $17 \mathrm{a}$ & $18 b$ & 19 & 20 & 21 & 22 \\
\hline & & & & & & & & & \\
\hline Sin respuesta & 0 & 1 & 3 & 0 & 1 & 2 & 3 & 23 & 4 \\
\hline Correctas & 5 & 20 & 17 & 15 & 11 & 4 & 25 & 3 & 17 \\
\hline Incorrectas & 30 & 14 & 15 & 20 & 23 & 29 & 7 & 9 & 14 \\
\hline Uso de las letras & & & & & & & & & \\
\hline Evaluada & 0 & 4 & 0 & 6 & 0 & 5 & 0 & 0 & 0 \\
\hline Ignorada & 0 & 5 & 0 & 0 & 0 & 0 & 0 & 0 & 0 \\
\hline Como objeto & 4 & 0 & 11 & 14 & 23 & 0 & 4 & 0 & 10 \\
\hline Incógnita de valor especifico & 26 & $\checkmark$ & 0 & 0 & 0 & 16 & 0 & $\checkmark$ & 0 \\
\hline Número generalizado & $\checkmark$ & 0 & $\checkmark$ & $\checkmark$ & $\checkmark$ & 0 & & 0 & $\checkmark$ \\
\hline Variable & 0 & 0 & 0 & 0 & 0 & $\checkmark$ & 0 & 0 & 0 \\
\hline Otros & 0 & 5 & 4 & 0 & 0 & 8 & 3 & 9 & 4 \\
\hline
\end{tabular}

En este caso hacemos especial énfasis en las tareas 3 y 19, las cuales presentan la mayor frecuencia de respuestas erróneas entre los estudiantes ubicados en el nivel 3. Estas dos tareas son las de más alta exigencia cognitiva, ya que para responderlas de manera correcta los estudiantes deben de involucrar la comprensión del uso y significado de las letras como números generalizados y como variable. Para la tarea 3, el $87.5 \%$ de los estudiantes expresaron una respuesta incorrecta, mientras que casi el $83 \%$ contestaron de manera incorrecta la tarea 19. En ambos casos se advirtió que la mayoría de los estudiantes, tienden a emplear las letras como incógnitas de valor específico. Estos registros altos en las respuestas incorrectas, nos corroboran la falta de conocimientos de los estudiantes de este nivel para el manejo y comprensión de las letras como números generalizados y como variables; así mismo, se encontraron bastantes evidencias de estudiantes, que a pesar de estar ubicados dentro de uno de los niveles de mayor exigencia cognitiva en el manejo de las letras, continúan con su tendencia a utilizar las letras como objetos, como se identificó en las respuestas encontradas en el ítem 18 (b) (Figura 3).

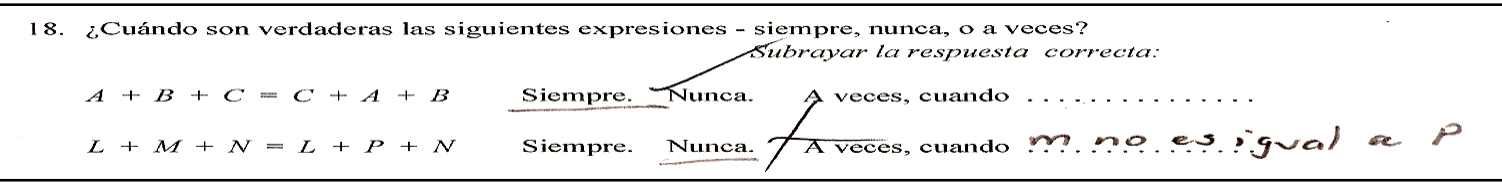

Figura 3 - Respuestas a la cuestión 18 del estudiante número 176 
Esta respuesta concuerda con un patrón repetitivo de soluciones a este ítem. Específicamente 20 de los 35 estudiantes ubicados en este nivel dieron como respuesta Nunca, lo que nos llevó a suponer que la fuente del error que ocasionaba las respuestas incorrectas podría ser que los estudiantes consideraban las letras como objetos o que letras distintas no pueden tener valores iguales.

Otro tipo de respuestas comunes, son las que encontramos para la tarea 3 . La figura 4 muestra un ejemplo de respuesta dada a esta tarea.

3. Cuál es más grande, $2 n$ o $n+2 \quad n+2$.

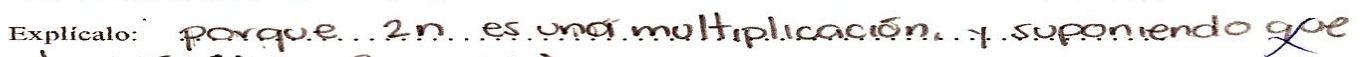
$n=1$ entonces $1+2=3$ y $2(1)=2$

Figura 4 - Respuestas a la tarea 3 del estudiante número 190

Cómo se puede observar en la respuesta del estudiante, persiste su tendencia a utilizar las letras como incógnitas de valores específicos y no concebir que las letras pueden representar un rango de valores (número generalizado), limitándose a elegir un par de valores que satisfagan la expresión que se le presenta y dando como válido el resultado correspondiente.

Respecto a la tarea 19 , casi el $75 \%$ de los estudiantes ubicados en este nivel proporcionaron respuestas similares a la ilustrada en la figura 5.

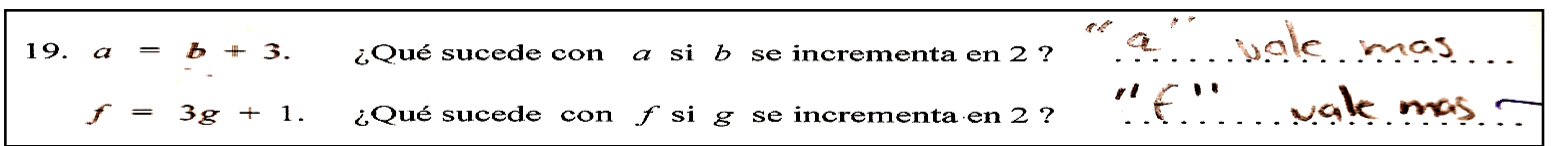

Figura 5 -Respuesta a la tarea 19 del estudiante 189

Los resultados obtenidos muestran que sólo un poco más del $11 \%$ de los estudiantes ubicados en el nivel 3 fueron capaces de emplear las letras como variables. Más de la mitad de los estudiantes del mismo nivel emplearon el uso de la letra como incógnita de valor especifico, como procedimiento alternativo para resolver este ítem.

Conforme a las evidencias encontradas en el análisis de las respuestas en este nivel de entendimiento, la mayoría de los estudiantes presentó carencias significativas en el uso y comprensión del significado de las letras como números generalizados y como variable. 


\subsection{Resultados particulares del nivel 4 de entendimiento}

Para la ubicación de los estudiantes en el nivel de mayor exigencia cognitiva, consideramos aquellos que eran capaces de emplear las 6 categorías descritas del uso de las letras. Los resultados obtenidos del análisis de las respuestas, nos indicaron que el $2.7 \%$ del total de la muestra quedaron ubicados en este nivel de acuerdo a los criterios establecidos en el Cuadro 1. En la tabla 6 se muestran los resultados de los alumnos ubicados en este nivel respecto a los ítems correspondientes al mismo.

Tabla 6 - Estudiantes del nivel 4: tipos de respuesta y usos de las letras

\begin{tabular}{lccccccccc}
\hline \multirow{2}{*}{$\begin{array}{l}\text { Tipo de respuesta } \\
\text { Sin respuesta }\end{array}$} & 3 & $4 \mathrm{e}$ & $7 \mathrm{~d}$ & $17 \mathrm{a}$ & $18 \mathrm{~b}$ & 19 & 20 & 21 & 22 \\
\cline { 2 - 9 } Correctas & 0 & 0 & 0 & 0 & 0 & 1 & 0 & 0 & 0 \\
Incorrectas & 1 & 5 & 5 & 4 & 4 & 1 & 5 & 4 & 5 \\
\hline Uso de las letras & 4 & 0 & 0 & 1 & 1 & 3 & 0 & 1 & 0 \\
Evaluada & 4 & 0 & 0 & 0 & 0 & 0 & 0 & 1 & 0 \\
Ignorada & 0 & 0 & 0 & 0 & 0 & 0 & 0 & 0 & 0 \\
Como objeto & 0 & 0 & 0 & 1 & 1 & 0 & 0 & 0 & 0 \\
Incógnita de valor especifico & 0 & $\checkmark$ & 0 & 0 & 0 & 3 & 0 & $\checkmark$ & 0 \\
Número generalizado & $\checkmark$ & 0 & $\checkmark$ & $\checkmark$ & $\checkmark$ & 0 & 0 & 0 & $\checkmark$ \\
Variable & 0 & 0 & 0 & 0 & 0 & $\checkmark$ & 0 & 0 & 0 \\
\hline
\end{tabular}

Es importante enfatizar que los estudiantes situados en este nivel muestran avances significativos en cuanto a la comprensión de los diferentes usos y significados de las letras, pues la mayoría no deja sin respuesta ninguna de las tareas que le exigen un menor nivel de dificultad cognitiva. Otra evidencia de su mayor nivel cognitivo se manifestó al no haber encontrado respuestas incorrectas relacionadas con la omisión de las letras presentes en las tareas. Sin embargo, el análisis de las respuestas a las tareas 3 y 19, nos arrojan resultados poco satisfactorios relacionados con la comprensión y manejo de las letras como números generalizados y variables, ya que casi la totalidad de los estudiantes fueron incapaces de resolver de manera correcta la tarea 3, la cual implicaba una respuesta por medio de una expresión que inducía a la generalización del resultado. Otro aspecto a destacar, es el hecho de que sólo un estudiante ubicado en el nivel 4, fue capaz de contestar de manera correcta la tarea 19 que, como se mencionó anteriormente, estaba diseñada para propiciar que los 
estudiantes demostraran su capacidad para emplear y comprender el significado de las letras como variables. Así pues, sólo 5 estudiantes de los 194 de la muestra, el 2.7\%, se sitúan en el nivel 4.

\subsection{Resultados generales por niveles de entendimiento}

La presente investigación permite vislumbrar de manera global dos resultados preliminares. El primero, que el cuestionario y las entrevistas han permitido conocer y caracterizar el bajo nivel de conocimientos algebraicos que los estudiantes tienen al ingresar a la Universidad, a través de los errores más comunes que han quedado evidenciados en esta investigación. La segunda es que los errores evidenciados en este estudio no corresponden al grado académico de los estudiantes. A continuación se fundamentan cada uno de ellos.

Con base a los resultados del análisis del total de respuestas de la prueba y a la clasificación de los estudiantes en los distintos niveles de entendimiento, reconocemos que en general todos los estudiantes ingresan con un bajo nivel algebraico como se muestran en la tabla 7.

Tabla 7 - Resultados generales por nivel de entendimiento

\begin{tabular}{ccc}
\hline Nivel de comprensión & Número de alumnos & $\%$ \\
\hline Nivel 0 & 0 & 0 \\
Nivel 1 & 50 & 25.6 \\
Nivel 2 & 105 & 53.8 \\
Nivel 3 & 35 & 17.9 \\
Nivel 4 & 5 & 2.7 \\
\hline
\end{tabular}

Como se puede observar en la tabla 7, no encontramos evidencias de estudiantes que presentaran deficiencias en sus conocimientos algebraicos que los ubicaran en el nivel 0 . Sin embargo, sí identificamos 50 estudiantes universitarios $(25,6 \%)$ que fueron ubicados en el nivel 1, ya que manifestaron un aceptable manejo de las letras al evaluarlas y las identificaron como objetos con valores propios. Sin embargo, en ocasiones ignoraron su presencia en las expresiones algebraicas y fueron incapaces de identificarlas con otro significado. Por otro lado, 104 estudiantes $(53,8 \%)$ mostraron, además de las habilidades propias del nivel 1 , evidencias de, al menos, identificar las letras como incógnitas de valor específico. Pero al igual que los anteriores, tampoco fueron capaces de comprender el uso de las letras como números generalizados o variables. 
Con estos resultados, corroboramos que la mayor parte de los estudiantes de nivel universitario que participan en esta investigación (79,4\%), a pesar de haber tomado cursos previos de algebra en los niveles educativos precedentes, poseen conocimientos algebraicos deficientes. También cabe señalar que 35 estudiantes manifestaron evidencias del manejo de las letras como números generalizados, lo que permitió ubicarlos en el nivel 3, una vez comprobadas sus competencias en la comprensión del uso y significado de las letras exigidos para superar los niveles inferiores. Debemos destacar que tan sólo 5 estudiantes se ubicaron en el nivel 4 de entendimiento, mostrandose una notable comprensión y manejo del uso y significado de las letras en sus más elevados niveles cognitivos. Finalmente, debemos mencionar que sólo un estudiante fue capaz de contestar de manera correcta el ítem 19, el único diseñado para ser resuelto por medio del uso y comprensión del significado de las letras como variables.

Este análisis nos brinda evidencias de que, aproximadamente, el $80 \%$ de los estudiantes sólo son capaces de reconocer el uso de las letras evaluadas, letras como objetos y letras como incógnitas con valor específico. Además, se detectó la recurrencia de ignorar, en una gran cantidad de respuestas de estos alumnos, la presencia de las mismas en las expresiones algebraicas que se les presentaron.

Por otra parte, sólo algo más del $20 \%$ de los estudiantes fueron capaces de identificar el uso de las letras como números generalizados: sólo cinco estudiantes mostraron evidencias parciales del uso de las letras como variables. Así mismo, sólo un estudiante fue capaz de responder, al mismo tiempo y de manera correcta a las tareas 3 y 19 diseñadas para evaluar el manejo de las letras como números generalizados y como variables.

De los resultados obtenidos en las pruebas, así como en las entrevistas semiestructuradas realizadas, deducimos que esos errores no se corresponden al nivel educativo donde desarrollamos este estudio: los errores encontrados en las pruebas que realizó Küchemann (1980) con estudiantes de secundaria, aún prevalecen en las producciones de los estudiantes universitarios participantes en este estudio.

Los resultados obtenidos en este estudio sugieren que durante el paso de los cursos de matemáticas de los estudiantes, previos a su ingreso en la universidad, no adquieren conocimientos necesarios para ser capaces de concebir los distintos usos de las letras en álgebra. En consecuencia, no alcanzan a desarrollar un aprendizaje significativo global, en el que el significado de variable no se desarrolla, si bien se considera necesario en estudios universitarios que incluyan materias de álgebra. 


\section{Reflexiones finales}

Partiendo de una situación problemática constatada por la literatura de investigación sobre el rendimiento en álgebra de estudiantes universitarios, hemos adoptado un instrumento elaborado por Küchemann (1980) que ha resultado apropiado para lograr nuestros objetivos, si bien ha resultado imprescindible adaptarlo al contexto mexicano. Los resultados de su aplicación nos han permitido analizar algunas de las causas de errores y los niveles de rendimiento de los sujetos analizados.

Destacamos que el total de los estudiantes participantes en esta investigación manifestaron respuestas incorrectas en la prueba aplicada. No obstante, no hubo evaluaciones erradas en su totalidad de los ítems para ninguno de los estudiantes; se constató que si bien existe capacidad en los estudiantes universitarios para la comprensión del uso de las letras en distintos contextos, no es la esperada en ese nivel educativo. Especialmente esto se ve refrendado en las respuestas incorrectas de la tarea diseñada, para evaluar el uso de la letras como variables: casi la totalidad de los estudiantes la contestaron de manera errónea y esto nos hace pensar que no comprenden ese significado de las letras.

Parece pues conveniente establecer líneas de apoyo encausadas para coadyuvar a los estudiantes universitarios a superar las dificultades que representan los diferentes usos de las letras en el álgebra. También parece necesario revisar con detalle el tratamiento que recibe el álgebra en los programas de estudio de los niveles de primaria, secundaria y bachillerato en cuanto al significado de las letras y especialmente en su papel de variable. Podría tambien ser interés, atender a la formación de profesores acerca del tratamiento escolar que deben tener los diferentes usos de las letras, para de esta forma, tratar de lograr un desarrollo más adecuado del pensamiento algebraico de los estudiantes.

\section{Referencias}

BACKHOFF, E. y TIRADO, F. Habilidades y Conocimientos Básicos del Estudiante Universitario: hacia los estándares nacionales. Revista de la Educación Superior, México, D.F, v. 22, n. 88, p. 4565, Diciembre. 1993.

BOOTH, L. R. Child methods in secondary mathematics. Educational Studies in Mathematics, Queensland, v. 15, n.12, p. 29-41, Feb. 1981.

BOOTH, L. R. Children's difficulties in beginning algebra. In: A. F. Coxford y A.P. Shulte (Ed.). The Ideas of Algebra, K-12.Reston, VA: National Council of Teachers of Mathematics, 1988. p. 20-32. 
CAPUTO, S. y MACIAS, D. Análisis de los errores de los alumnos de la asignatura. "Algebra I" al elaborar demostraciones. En: Reunión anual de Comunicaciones Científicas de la Unión Matemática Argentina. 41., 2006, Buenos Aires, Argentina. Disponible en:

<http://www.unne.edu.ar/unnevieja/Web/cyt/cyt2006/09-Educacion/2006-D-012.pdf > Acesso en: 11 dic. 2012.

COLLIS, K. F. A study of concrete and formal operations in school mathematics: A Piagetian viewpoint, Melbourne: Australian Council for Educational Research, 1975.

DAVIS, R. B. Cognitive Processes Involved in Solving Simple Algebraic Equations. Journal of Children's Mathematical Behavior, New Jersey, v.1, n. 3, p. 7-35, 1975.

FERREYRA,N.; RECHIMONT, E.; PARODI, C.; CASTRO, N. De la aritmética al álgebra. Experiencia de trabajo con estudiantes universitarios. UNION.Revista Iberoamericana de Educación Matemática, La laguna, n. 21, p. 59-67,Mar. 2010.

HERNÁNDEZ, R.; FERNÁNDEZ, C y BAPTISTA P.Metodología de la Investigación. México: McGraw Hill, 1999.

HODGEN, J., KÜCHEMANN, D., Brown, M., \& Coe, R. Children's understandings of algebra 30 years. Research in Mathematics Education, Reston, v. 11, n. 2, p. 193-194, Sep. 2009.

KIERAN, C. The Learning and Teaching of School Algebra. In: D. A. GROUWS (Ed.), Second Handbook of Research on Mathematics Teaching and Learning. New York: Information Age Publishing, 1990. p. 390-419.

KÜCHEMANN, D. Children's understanding of numerical variables. Mathematics in School, v. 7, n. 4, p. 23-26, Sep. 1978.

KÜCHEMANN, D. The understanding of generalised arithmetic (algebra) by secondary school children. 1980. 232h. Tesis (Doctoral en Filosofía) sin publicar,University of London, London, 1980.

LINCHEVSKI, L. Algebra with numbers and arithmetic with letters: a definition of pre-algebra. Journal of Mathematical Behavior, New Jersey, v. 14, n.1, p. 113-120, Mar. 1995.

MAC GREGOR, M. y STACEY, K. Students' understanding of algebraic notation: 11-15.Educational Studies in Mathematics, Queensland, v. 33, n.1, p. 1-19, Jun. 1997.

PALAREA M.M. La adquisición del lenguaje algebraico: reflexiones sobre una investigación. Números. Revista de didáctica de las matemáticas, Santa Cruz de Tenerife, v. 40, p. 3-28, Dic.1999.

PHILIPP, R. The many uses of algebraic variables. Mathematics Teacher, Reston, v. 85, n.7, p. 557561, Oct. 1992.

POCHULU, M. Análisis y categorización de errores en el aprendizaje de la Matemática en alumnos que ingresan a la Universidad. Revista Iberoamericana de Educación, Madrid, v. 35, n. 4, p.114,Mar. 2005.

RUANO, R. M.; SOCAS, M.; PALAREA, M. Análisis y clasificación de errores cometidos por alumnos de secundaria en los procesos de sustitución formal, generalización y modelización en álgebra. PNA, Granada, v. 2, n. 2, p. 61-74, Ene.2008. 
URSINI, S. y Trigueros, M.¿Mejora la comprensión del concepto de variable cuando los estudiantes cursan matemáticas avanzadas?, Revista Educación Matemática, México, D.F, v 18, n. 3, p. 5-38, Editorial Santillana. 2006.

URSINI, S. y TRIGUEROS, M. How do high school students interpret parameters in algebra? In: M. J. HOINES y A. B. FUGLESTAD (Ed.). Proceedings of the 28th Conference of the International Group for the Psychology of Mathematics Education. Norway: Bergen University College,2004. p. 361-368.

USISKIN, Z. Conceptions of school algebra and uses of variables. In: A. F. COXFORD. The Ideas of Algebra, K-12. Reston, VA: National Council of Teachers of Mathematics,1988. p. 8-19.

Submetido em Junho de 2013. Aprovado em Setembro de 2013. 